\title{
Diseño de una Arquitectura para la Ludificación de la Actividad Física en Niños
}

\author{
María Cosío ${ }^{1}$, Juan I. Nieto ${ }^{1}$, Mabel Vazquez ${ }^{1}$, Raymundo Buenrostro² ${ }^{2}$ Juan A. \\ Guerrero $^{2}$, Carlos A. Flores ${ }^{2}$ \\ ${ }^{1}$ Universidad Autónoma de Baja California \\ ${ }^{2}$ Universidad de Colima \\ ${ }^{1}$ \{cosio.maria, jnieto, mabel.vazquez\}@uabc.edu.mx, ${ }^{2}$ \{raymundo, antonio_guerrero, \\ cfcortes\}@ucol.mx
}

\begin{abstract}
Resumen. En este trabajo se presenta los resultados del diseño de una arquitectura para la ludificación de la actividad física, el objetivo de esta propuesta es proveer de desarrollos tecnológicos para generar hábitos en niños de realizar actividad física. Para ello se utilizan dos técnicas de la capa de red, el diseño de redes tolerantes al retraso y el concepto de enrutamiento oportunista; así como estándares de comunicación con dos alcances, el primero de muy corta distancia para identificar al individuo que realiza la actividad y otro de alcance mediano para alcanzar a objetos en cobertura que puedan hacer la retransmisión de los datos a internet. Adicional a estas dos técnicas, se propone el uso del concepto de interacciones embebidas para la activación de la acción con los objetos. Se describe también las características de la plataforma de juegos, principal incentivador en el espacio virtual para que los niños realicen la actividad. Se concluye con los escenarios para la simulación de la efectividad del diseño.
\end{abstract}

Keywords: WSN, redes DTN, ludificación, arquitectura.

\section{$1 \quad$ Introducción}

De las 1,200 millones de personas que tienen problemas de sobrepeso y obesidad en el mundo, 80 millones son mexicanos [1]. La obesidad infantil es actualmente un problema de salud en México, donde más de 4 millones de niños sufren de este problema, ello implica que 1 de cada 5 niños tiene problemas de sobrepeso y las tendencias en los hábitos para la realización de actividades físicas según lo muestran las estadísticas se reducen de forma considerable a través del tiempo [2], [3], [4].

La organización mundial de salud en su estrategia mundial sobre régimen alimentario, actividad fisica y salud tiene como objetivo general: Orientar a nivel local, nacional e internacional el desarrollo de actividades que, emprendidas conjuntamente, redundaran en mejoras del nivel de los factores de riesgo y reducirán las tasas de morbilidad y mortalidad de la población por enfermedades crónicas relacionadas con el régimen alimentario y la actividad física [1]. 
Es en el punto de las políticas o programas que permitan el incremento de las actividades físicas en los menores, que este trabajo propone hacer un aporte en la generación de algoritmos de comunicación, y de registro de las interacciones. La valoración de la actividad física se pretende realizar a través de objetos de uso común con capacidades de adquisición/transmisión de datos.

El problema a resolver es el siguiente: ¿Se puede motivar, usando la tecnología para que los niños realicen actividad física? considerando que los hábitos actuales respecto a las formas de juego se circunscriben a espacios cerrados y baja movilidad; formas que son ampliamente apoyadas por las personas encargadas de sus cuidados, derivado de una problemática como la que describe la Figura 1.

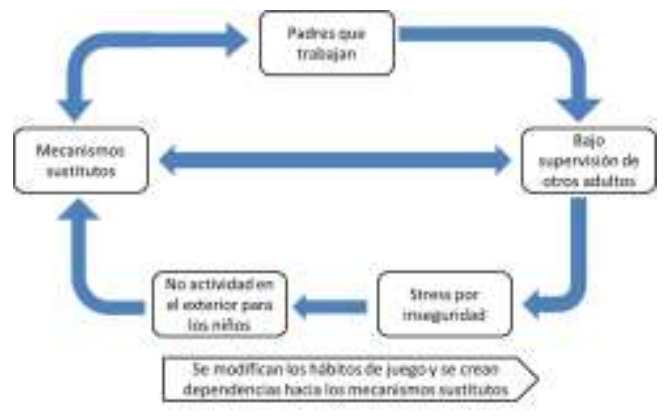

Figura 1. Planteamiento del problema

Para lograrlo se plantea diseñar una arquitectura de comunicaciones, con consciencia de la ubicación y de la fuente de datos (sujeto quien realiza la actividad física). Los nodos que conforman la red, se propone sean objetos que ejecuten tareas de encaminamiento, adquisición/transmisión de datos. Los nodos, pueden o no en conjunto ofrecer una ruta en tiempo real a Internet; la anterior condición debido a restricciones en la conectividad, que pueden existir en el lugar donde se realice la actividad física.

Principales contribuciones en este trabajo son: a) el diseño de una arquitectura de red que permite cuantificar la actividad física, identificar a quien la realiza y la interconexión con plataformas de juegos abiertas; b) una plataforma de juego que usa valores relacionados con el nivel de actividad física como dinero electrónico para activarse.

\section{Trabajos Relacionados}

En la literatura se han publicado varios trabajos relacionados con el control de la obesidad mediante videojuegos. Ejemplo de soluciones tecnológicas son provistas por las grandes compañías como Wii, Kinetic entre otras. En el estado del arte propuestas como la de los autores en [13] llamada Healthy Weight Game!, da seguimiento mediante un avatar de como la persona pierde peso conforme realiza la actividad 
física, diferente de la propuesta presentada en este trabajo, existe una consciencia plena de quien lo usa respecto a su deseo de perder de peso, condición que es difícil lograr en menores de edad. En [14] se propone una evaluación del impacto del uso de juegos para generar hábitos en niños entre 10 y 12 años con resultados positivos en el uso de esta clase de propuestas. Una nueva generación de videojuegos llamados Active Video Games (AVG), se estudia en [15] mediante una exploración del estado del arte. Se concluye que son excelentes herramientas pero estos datos tienen una gran desventaja, ya que la mayoría son resultados de estudios de laboratorio, de ahí que lograr el diseño que aquí se propone podría proveer información para la investigación y como lo demuestran los anteriores trabajos citados afectar de forma positiva los hábitos de los niños respecto a la actividad física.

\section{Descripción de los modelos de la Arquitectura}

Analizando el problema a resolver, se visualiza un escenario con baja conectividad; condiciones con las que debe lidiar la arquitectura que se propone en este trabajo. Esta red tiene las características de una MANET (como acrónimo de Mobile ad hoc network) [5]; redes que puede generar islas de conectividad local sin acceso a Internet, compuestas por objetos móviles, como bicicletas, material deportivo personal, teléfonos celulares pertenecientes a personas con patrones de traslado bien definidos, hacia zonas que permiten conectividad a Internet. Integrando lo anterior procedemos a describir en la siguiente subsección el modelo de red.

\subsection{Modelo de red}

El modelo de red que propone involucra el paradigma del Internet de las Cosas (IoT); este es un paradigma asociado a las comunicaciones inalámbricas. El concepto básico es la presencia pervasiva de objetos o cosas comunes habilitados con tecnología que permite la adquisición y transmisión de información del contexto a Internet. Este paradigma asocia tecnologías de comunicaciones como identificadores de radio frecuencia (RFID), dispositivos de adquisición de datos, actuadores, entre otras. Los objetos usan direcciones únicas para interactuar y cooperar [6]. Con el anterior marco se propone añadir una característica adicional, una jerarquía en la organización de los nodos, que se define considerando la aplicación de los datos [7], las capacidades de conectividad de los objetos y su uso, la estructura se muestra en la Figura 2.

En el nivel inferior de la estructura se ubican los nodos móviles, estos dispositivos una vez que tienen en cobertura a un nodo pasarela, iniciaran a transmitir paquetes para solicitar se registre su interacción con la red. El resto de los niveles están constituidos por dispositivos pasarela, estáticos o móviles, considerando en la jerarquía más alta, el dispositivo pasarela que permite conectividad a internet, cada uno de los elementos en la arquitectura son descritos en la Tabla 1. 


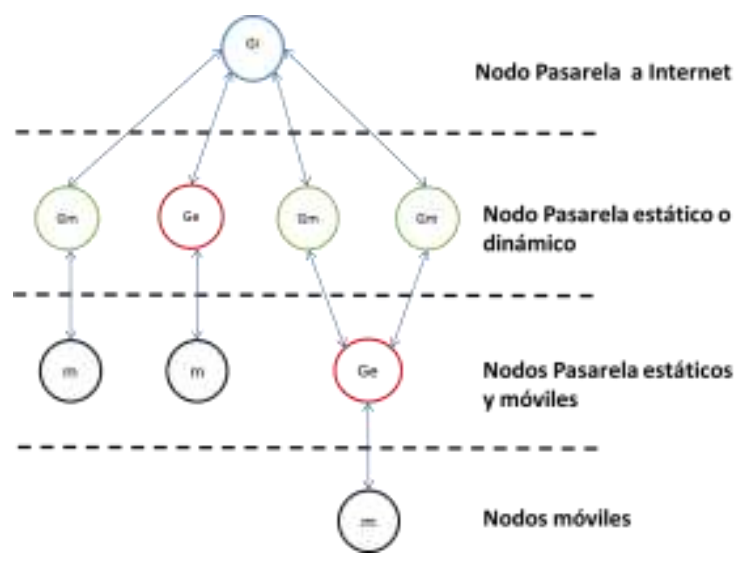

Figura 2. Estructura Jerárquica.

Tabla 1. Descripción de los nodos considerados en la red.

\begin{tabular}{|c|c|}
\hline NODO & DESCRIPCION \\
\hline $\begin{array}{l}\text { Dispositivo } \\
\text { Móvil [m] }\end{array}$ & $\begin{array}{l}\text { Dispositivo portado por el usuario final niños entre } 6 \text { y } 11 \text { años, éste } \\
\text { cuenta con un radio de comunicación IEEE } 802.15 .4 \text {, configurado } \\
\text { para una cobertura es de } 10 \text { a } 50 \text { cms. Su tarea es la transmisión de } \\
\text { paquetes que incluyen un identificador único. Este intercambio de } \\
\text { datos inicia cuando el dispositivo móvil encuentra un nodo pasarela } \\
\text { al cual asociarse, su función es la activación de las interacciones } \\
\text { embebidas en el dispositivo destino. Este dispositivo no puede } \\
\text { interactuar con dispositivos de su nivel jerárquico. }\end{array}$ \\
\hline $\begin{array}{l}\text { Pasarela } \\
\text { estático [Ge] }\end{array}$ & $\begin{array}{l}\text { Dispositivo con dos interfaces de red, IEEE } 802.15 .4 \text { e IEEE } \\
802.11 \text { b. La capacidad de almacenamiento se propone de } 512 \text { Bytes. } \\
\text { Para la retransmisión de los datos adquiridos se comunica a un } \\
\text { dispositivo pasarela estático o móvil con conectividad a Internet o } \\
\text { no. Este dispositivo contendrá información de paquetes reenviados } \\
\text { y paquetes perdidos debido a la condición de buffer saturado. }\end{array}$ \\
\hline $\begin{array}{l}\text { Pasarela } \\
\text { móvil }[\mathrm{Gm}]\end{array}$ & $\begin{array}{l}\text { Estos d son habilitados con dos interfaces, IEEE } 802.15 .4 \text { e IEEE } \\
802.11 \text { b. Con capacidad de almacenamiento de } 512 \text { Bytes. Éste } \\
\text { almacenará información de la cantidad de dispositivos con } \\
\text { conectividad a Internet con los que ha interactuado, paquetes } \\
\text { descartados debido a la saturación de pila y paquetes entregados; } \\
\text { datos que son enviados de forma continua al servicio administrador } \\
\text { de objetos en Internet. }\end{array}$ \\
\hline $\begin{array}{l}\text { Pasarela a } \\
\text { Internet [Gi] }\end{array}$ & $\begin{array}{l}\text { Dispositivo con una interfaz IEEE } 802.11 \mathrm{~b} \text {, éste es el máximo nivel } \\
\text { dentro de la arquitectura de transmisión de datos, ya que dirige el } \\
\text { flujo de datos hasta el servidor de juegos en Internet. }\end{array}$ \\
\hline
\end{tabular}




\subsection{Enrutamiento oportunista}

Como ya se definió, las redes ad-hoc permiten la comunicación entre grupos de dispositivos móviles sin la necesidad de una infraestructura de red preexistente. Una interesante evolución de este tipo de redes, son las redes oportunistas (OppNets); el objetivo de esas redes es habilitar las comunicaciones entre dispositivos donde no existe una conexión End-to-End entre el transmisor y el receptor de forma segura [8]. En estos escenarios los datos deben ser entregados basado en oportunidades de hacer parejas; paradigma considerado para interconectar objetos que requieran realizar transmisiones a Internet [9]. Los requerimientos de transmisión de datos en los dispositivos surgen debido a la interacción entre humanos y los dispositivos del IoT, para generar una reacción en el espacio virtual, ante una interacción física.

\subsection{Interacciones embebidas}

En [10] los autores definen interacción embebida, como la perfecta integración para la interacción entre los fenómenos tecnológicos y los conceptuales de los artefactos del día a día. Técnicamente, esto requiere que los objetos comunes cuenten propiedades como las que caracterizan a los del paradigma del IoT. Conceptualmente, se requiere que se den interacciones en las tareas del día a día de los usuarios. Adicional a las características antes mencionadas, los objetos pueden tener funcionalidades embebidas, las cuales puedan activarse mediante interacciones que se den con otros objetos en el mismo espacio físico [11]. Con esta filosofía, se propone el uso de objetos comunes, como bicicletas, pelotas, etc. que nos permitan monitorizar la actividad; para convertirla en moneda para la adquisición de tiempo en video juegos.

\subsection{Modelo de Datos}

En este modelo se cuenta con cuatro hitos en la transferencia de datos: servidor de juegos, sockets de entrada, el $\mathrm{Gm}$ y el juguete con interacciones embebidas. El modelo de datos en la arquitectura de monitorización se propone unidireccional, esto significa que no se cuenta con mecanismos que permitan asegurar la recepción de los datos en el destino. Existen dos razones de este diseño, la primera es una fuente de datos de bajo costo (dispositivo portado por los niños), por lo tanto no se contempla gran capacidad de almacenamiento. La segunda asociada a las características del modelo de red; DTN (Delay-tolerant Networking) [12], usando enrutamiento oportunista [8] para la transmisión de datos entre objetos.

En el servidor de juegos se propone utilizar el diseño de base de datos mostrado en la Figura 3; en este diseño se consideran dos tipos de objetos, los reales y los virtuales; los primeros se refieren a los objetos con los cuales los niños realicen la actividad física y los segundos son aquellos que existen en la plataforma de juegos, que se 
describe en la siguiente sección.

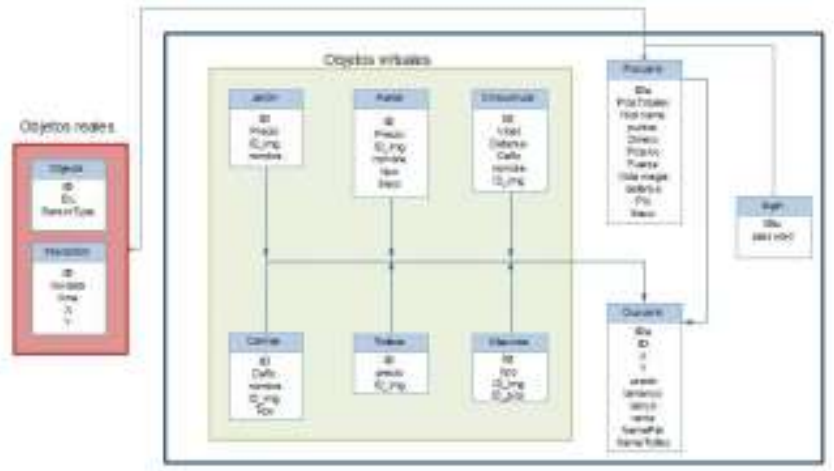

Figura 3. Base de datos

\section{$4 \quad$ Plataforma de Juegos}

La plataforma de juegos desarrollada se clasifica como de micro transacciones, en el cual los jugadores pagan por implementos adicionales; en el caso del juego propuesto, esta tarea es posible realizarla en el módulo Tienda; en ella por ejemplo, se puede comprar hechizos, armas, escudos, entre otros elementos; ver detalle en la Figura 4 donde se muestra cada uno de los elementos que conforman el juego.

En el módulo Carrera, se cuenta con un caso especial de interacción, ya que abre la oportunidad para quien pertenezca a un club pueda de esa forma obtener dinero adicional o la capacidad de hacer compras. El mecanismo para hacerlo implica que si en el club gano un trofeo por alguna actividad o alcanzó algún éxito relevante, se refleje en la plataforma de juegos. Para lograrlo el club debe solicitar el socket que le permita interactuar con el servidor de base de datos.

\section{Diseño de los escenarios de red para evaluación}

El objetivo en este punto es describir los escenarios de red que se proponen para la simulación, y evaluación de los algoritmos propuestos considerando: las áreas donde realizan actividad física con mayor frecuencia los menores, estos son espacios claramente delimitados y muchos de ellos con conectividad a Internet. Se propone para el caso de uso una escuela primaria, ya que esta clase de espacios nos permite tener diversos escenarios y niveles de actividad física. Más aún, para las pruebas insitú ofrece la ventaja de que los dispositivos de adquisición de datos pueden ser resguardados, para realizar la monitorización de la actividad por largos periodos. 


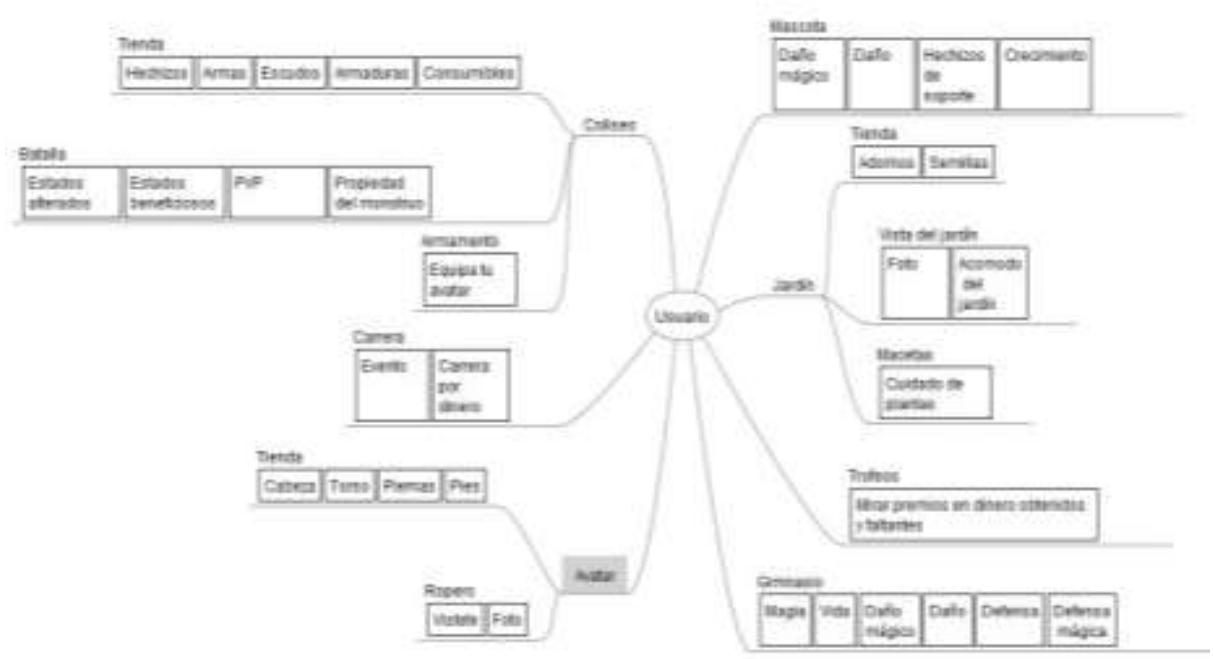

Figura 4. Mapa mental del diseño de la plataforma de juegos

Para definir lo anterior se visitaron dos escuelas y dos parques, ambas escuelas de la zona urbana; respecto a los parques uno de ellos era en la zona rural. En una de las escuelas se observó en una mañana como los alumnos se distribuían en las áreas comunes durante su tiempo de descanso y algunas actividades programadas durante una clase de educación física. Derivado de estas observaciones se proponen tres escenarios: (a) Cancha de baloncesto, (b) El área de juegos fijos como resbaladillas, columpios y pasamanos, (c) áreas de jardines y finalmente (d) área de asta bandera que permite el juego libre

Adicional a esta información se preguntó por la conectividad a Internet, la escuela si contaba con ese acceso pero solo en tres aulas cercanas a la dirección. En estas aulas se imparten clases a alumnos de 5to. y 6to. año; la tercer aula es donde se ubican las oficinas de la dirección, con lo cual constatamos las condiciones de conectividad limitadas. Con esta información preliminar se definieron los requerimientos funcionales y no funcionales de la arquitectura de comunicaciones, información que sirvió de insumo para el diseño de los escenarios de simulación y los algoritmos.

\section{Conclusiones}

En este trabajo presentamos la propuesta de una arquitectura que permita la ludificación de la actividad física en niños. Este trabajo plasma el diseño completo de la arquitectura de ludificación que se refleja en la definición de las entidades funcionales, los protocolo de comunicación y de interacción entre los objetos y los 
individuos, esto último con el objetivo de cerrar el ciclo para relacionar la actividad física y los videos juegos. Se concluye este documento con las consideraciones iniciales para el escenario de evaluación del modelo de red. Dejándose como trabajo a futuro las tareas de simulación, diseño de dispositivos y la implementación de los protocolos en dichos dispositivos.

\section{Referencias}

1. WHO: Guía para incrementar los niveles de actividad física. (2008)

2. Hospital Infantil de México Federico Gomez Dr. Marquez No.162, Col. Doctores, Delegación: Cuauhtémoc México D.F. C.P 06720 - Tel. 5228 9917: (GUIA DEL ABORDAJE DIAGNSTICO Y DE MANEJO DE LA OBESIDAD INFANTIL)

3. Quezada, J.P.A.: El reto de la obesidad infantil en México. Centro de Centro de Sociales y de Opinión pública (2012)

4. de Salud pública, I.N.: Encuesta nacional de salud y nutrición 2012, resultados nacionales. Technical report, Secretaria de Salud (2012)

5. Awerbuch, B., Holmer, D., Rubens, H.: The medium time metric: High throughput route selection in multi-rate ad hoc wireless networks. Mob. Netw. Appl. 11 (2006) 253-266

6. Atzori, L., Iera, A., Morabito, G.: The internet of things: A survey. Comput. Netw.

54 (2010) 2787-2805

7. Psaras, I., Wood, L., Tafazolli, R.: Delay-disruption-tolerant networking: State of the art and future challenges, Center for Communication Systems Research (CCSR), Dept. of El. Eng., University of Surrey Guildford, GU2 7XH, Surrey, UK Surrey, England (2009)

8. Karamshuk, D., Boldrini, C., Conti, M., Passarella, A.: Human mobility models for opportunistic networks. Communications Magazine, IEEE 49 (2011) 157-165

9. Daly, E.M., Haahr, M.: Social network analysis for routing in disconnected delay- tolerant manets. In: Proceedings of the 8th ACM International Symposium on Mobile Ad Hoc Networking and Computing. MobiHoc '07, New York, NY, USA, ACM (2007) 32-40

10.Kranz, M., Holleis, P., Schmidt, A.: Embedded interaction: Interacting with the internet of things. Internet Computing, IEEE 14 (2010) 46-53

11.Guo, B., Zhang, D., Yu, Z., Liang, Y., Wang, Z., Zhou, X.: From the internet of things to embedded intelligence. World Wide Web 16 (2013) 399-420

12.Massri, K., Vernata, A., Vitaletti, A.: Routing protocols for delay tolerant net- works: A quantitative evaluation. In: Proceedings of the 7th ACM Workshop on Performance Monitoring and Measurement of Heterogeneous Wireless and Wired Networks. PM2HW2N '12, New York, NY, USA, ACM (2012) 107-114

13.Lentelink, S.J.; Spil, AAM.; Broens, T.; Hermens, H.J.; Jones, V.M., "Healthy weight game!: Lose weight together," Serious Games and Applications for Health (SeGAH), 2013 IEEE 2nd International Conference on , vol., no., pp.1,8, 2-3 May 2013 doi: 10.1109/SeGAH.2013.6665311.

14.do Carmo, Joao; Goncalves, Ricardo; Batalau, Rui; Guilherme, Jose; Palmeira, Antonio L., "Active video games in schools to enhance children's physical activity," Serious Games and Applications for Health (SeGAH), 2013 IEEE 2nd International Conference on, vol., no., pp.1,4, 2-3 May 2013, doi: 10.1109/SeGAH.2013.6665320

15.do Carmo, Joao; Goncalves, Ricardo; Batalau, Rui; Palmeira, Antonio L., "Active Video Games and Physical Activity in overweight children and adolescents," Serious Games and Applications for Health (SeGAH), 2013 IEEE 2nd International Conference on , vol., no., pp.1,5, 2-3 May 2013 doi: 10.1109/SeGAH.2013.6665323 